\title{
Secondary magnetic field harmonics dependence on vacuum beam chamber geometry
}

\begin{abstract}
S. Y. Shim, ${ }^{*}$ S. Wilfert, and C. Muehle
GSI Helmholtzzentrum für Schwerionenforschung GmbH, Planckstraße 1, D-64291 Darmstadt, Germany (Received 30 January 2012; revised manuscript received 13 December 2012; published 26 August 2013)

The harmonic magnetic field properties due to eddy currents have been studied with respect to the geometry of the vacuum beam chamber. We derived a generalized formula enabling the precise prediction of any field harmonics generated by eddy currents in beam tubes with different cross-sectional geometries. Applying our model to study the properties of field harmonics in beam tubes with linear dipole magnetic field ramping clearly proved that the circular cross section tube generates only a dipole field from eddy currents. The elliptic tube showed noticeable magnitudes of sextupole and dipole fields. We demonstrate theoretically that it is feasible to suppress the generation of the sextupole field component by appropriately varying the tube wall thickness as a function of angle around the tube circumference. This result indicates that it is possible to design an elliptical-shaped beam tube that generates a dipole field component with zero magnitude of sextupole. In a rectangular-shaped beam tube, one of the selected harmonic fields can be prevented if an appropriate wall thickness ratio between the horizontal and vertical tube walls is properly chosen. Our generalized formalism can be used for optimization of arbitrarily complex-shaped beam tubes, with respect to suppression of detrimental field harmonics.
\end{abstract}

DOI: 10.1103/PhysRevSTAB.16.082401

\section{INTRODUCTION}

The required vacuum level and the minimizing of the magnetic field influence have to be considered in the design of the particle beam vacuum tubes. Because of these requirements, its material properties and geometrical shape are important parameters to achieve mechanical strength, vacuum, and field quality. The Facility for Antiproton and Ion Research (FAIR) project will have a $1.9 \mathrm{~T}$ maximum field, with $4 \mathrm{~T}$ / sec linear ramping dipole superconducting magnets. At these low operating temperatures, it is obviously advantageous to use the inner surface of the beam tube as a highly effective cryopump in order to generate beam vacuum pressures in the range of $\sim 10^{-12}$ mbar. However, due to the fast ramping cycles, eddy current effects will be rather intense in the dipole tubes. This leads not only to a heating of the tube walls, but also causes field inhomogeneities in the form of multipoles. These secondary effects may not only negatively affect the pumping properties of the cold tubes but can also influence the beam life time and even cause beam losses [1]. In order to improve the magnet performance, extensive experiments and theoretical studies have been carried out on a superconducting magnet system and cryogenic heat losses including the beam tube. [2-5].

Several articles on eddy current profile calculations for various beam tube shapes and their corresponding

\footnotetext{
*Corresponding author. S.Y.Shim@gsi.de

Published by the American Physical Society under the terms of the Creative Commons Attribution 3.0 License. Further distribution of this work must maintain attribution to the author(s) and the published article's title, journal citation, and DOI.
}

PACS numbers: 07.55.Db, 41.20.Gz, 41.85.Lc, 85.70.Ay

magnetic fields have been published [2-9]. Most of the publications only deal with the typical and commonly used beam tube shapes, i.e., circular, elliptical, or rectangular. However, up to now a generally valid formula for computation of eddy current-induced multipole generation caused by arbitrarily shaped beam tubes is nonexistent. Motivated by the need to design dipole beam tubes with small eddy current effects for a dipole magnet, we theoretically analyzed the origin of eddy current-induced multipoles with respect to the beam pipe geometry. Hereby a generalized expression for the prediction of any harmonics caused by different cross-sectional shapes of beam tubes was found.

Based on our formalism, we analytically studied the contribution to field harmonics from beam tubes with typical cross sections, i.e., circular, elliptical, and rectangular, in time-varying magnetic fields. A method is discussed for the elimination of a single selected harmonic term by modifications of the tube geometry.

\section{MULTIPOLE FIELDS BY EDDY CURRENT}

Multipole coefficients will be written as functions of the beam tube geometry, allowing us to directly calculate the multipoles without field fitting or other numeric procedures. We consider a two-dimensional cross section of a beam tube that is located in the region of the magnet center. Neglecting magnet end effects and assuming a symmetric tube geometry, the eddy current will also have a symmetric current profile during the uniform magnetic field ramping. For dipole field symmetry, there is polarity of $I(x, y)=$ $I(x,-y)=-I(-x, y)=-I(-x,-y)$. Under this four line current symmetry, the corresponding magnetic field is given by $[10,11]$ 


$$
B_{\theta}(r, \theta)=-\frac{2 \mu_{0} I_{0}}{\pi a_{0}} \sum_{n=1,3,5, \ldots}^{\infty}\left[\left(\frac{r}{a_{0}}\right)^{n-1} \cos n \phi \cos n \theta\right],
$$

where $a_{0}$ and $\phi(0 \leq \phi \leq \pi / 2)$ are, respectively, the radius and angle of the current $I_{0}$ in a polar coordinate system. Equation (1) contains only odd-number harmonic fields $n=1,3,5, \ldots$ caused by the symmetric current flow that corresponds to dipole, sextupole, decapole, etc. [10].

With an infinite number of four symmetric line currents, we can decompose Eq. (1) for a certain harmonic $n$ as follows:

$$
\begin{gathered}
B_{\theta}(r, \theta)=\sum_{n=1,3,5, \ldots}^{\infty} B_{\theta, n}(r, \theta), \\
B_{\theta, n}(r, \theta)=-\frac{2 \mu_{0}}{\pi} \cos n \theta \sum_{i=1}^{\infty}\left[\left(\frac{I_{i}}{a_{i}}\right)\left(\frac{r}{a_{i}}\right)^{n-1} \cos n \phi_{i}\right] .
\end{gathered}
$$

Since the beam tube is continuous, the eddy current $I_{i}$ as well as the radius $a_{i}$ must be expressed in terms of a geometric variable. As both parameters $I_{i}$ and $a_{i}$ are angle dependent, we can change the notation to $I(\phi)$ and $a(\phi)$ for the current and radius. The $a(\phi)$ can be derived directly from the shape of a beam tube. In the case of a circular cross section, $a(\phi)$ corresponds simply to the radius or the distance from the coordinate system center. For an elliptical cross section, the radius can be expressed by $a(\phi)=$ $b / \sqrt{1-\varepsilon^{2} \cos ^{2} \phi}$, where $b$ is the minor axis and $\varepsilon$ represents the eccentricity of the ellipse. For other crosssectional geometries, $a(\phi)$ can be expressed analogously.

The profile of $I_{i}$ cannot be deduced simply from the beam tube geometry. A general procedure to get the profile of eddy current density $J_{i}$ does not exist since it is not only dependent on the applied field characteristics but also on the beam tube properties. The spatial profile of $J_{i}$ may show linear, nonlinear, or periodic shapes, depending on the applied field uniformity, frequency, sinusoidal behavior and linear/ nonlinear ramping. In addition, electrical and thermal conductor properties such as its inductance, conductance, and even heat capacitance at cryogenic temperature, can also affect the profile of the eddy current density $J_{i}$.

In this work, we consider the operational scenario of the SIS100 dipole, $4 \mathrm{~T} / \mathrm{sec}$ of linear field ramping with a repetition frequency of $1.2 \mathrm{~Hz}$. The skin depth effect is thus negligible. Under this linear ramping scenario, the induced emf is constant in time, and therefore, it induces a constant eddy current flow in the beam tube as well as a constant induced eddy current magnetic flux $\Phi_{\text {eddy }}$. The net emf can simply be expressed with applied magnetic flux $\Phi_{\text {app }}$ as $d \Phi_{\text {total }} / d t=d\left(\Phi_{\text {app }}+\Phi_{\text {eddy }}\right) / d t=d \Phi_{\text {app }} / d t$, which shows that $\Phi_{\text {app }}$ is the only source of eddy currents without iterative effect in the case of a uniform linear ramping field.

From the law of magnetic induction with uniform linear ramping field, the eddy current density $j$ can be expressed as $j=x \dot{B} / \rho$, where $\rho$ is resistivity and $x$ the horizontal coordinate. Note that this expression for $j$ accounts for information about the applied field and the material properties of the beam tube, while the information about the beam tube geometry is not included. Even though various shapes of eddy current conductor generate different field profiles, their eddy current density profiles are equal under the same applied field. The beam tube geometry-dependent eddy current profile can be expressed as a function of $\phi$ in polar coordinates as follows:

$$
d I(\phi)=j d A=\frac{\dot{B}}{\rho} R(\phi)^{2} D(\phi) \cos \phi d \phi,
$$

where $\quad R(\phi)=\left[a_{1}(\phi)+a_{2}(\phi)\right] / 2, \quad D(\phi)=a_{2}(\phi)-$ $a_{1}(\phi)$. The $a_{1}(\phi), a_{2}(\phi)$ are the inner and outer radii of the beam tube, respectively. Here $R(\phi)$ and $D(\phi)$ can be seen as the effective radius and the generalized thickness of the beam tube shell, respectively. The $D(\phi)$ is defined in a polar coordinate and not the same definition of the thickness perpendicular to the beam tube surface. Inserting Eq. (4) into Eq. (3) with geometry normalization $l(\phi)=$ $R(\phi) / R_{0}, \delta(\phi)=D(\phi) / D_{0}$ leads to the following expression for the eddy current-induced harmonic fields, where $R_{0}, D_{0}$ are the maximum value of the $R(\phi)$ and $D(\phi)$, respectively:

$$
\begin{aligned}
B_{\theta, n}(r, \theta)= & -2 R_{0} D_{0}\left(\frac{\mu_{0} \dot{B}}{\pi \rho}\right)\left(\frac{r}{R_{0}}\right)^{n-1} \cos n \theta \int_{0}^{\pi / 2} \frac{\delta(\phi)}{l(\phi)^{n-2}} \\
& \times \cos \phi \cos n \phi d \phi,
\end{aligned}
$$

where $r \leq R_{\min }, R_{\min }$ is the minimum beam tube radius. By finding an appropriate geometry of the beam tube that exhibits a constant ratio of $\delta(\phi)$ and $l(\phi)^{n-2}$, it becomes possible to eliminate certain harmonics of the eddy current-induced field. The geometry optimization will be continued in the next section.

Equation (5) can be written for the whole space as follows:

$$
\begin{aligned}
B_{\theta, n}(r, \theta)= & -2 R_{0} D_{0}\left(\frac{\mu_{0} \dot{B}}{\pi \rho}\right) \cos n \theta\left[\left(\frac{r}{R_{0}}\right)^{n-1} \int_{0}^{\Phi(r)} \frac{\delta(\phi)}{l(\phi)^{n-2}}\right. \\
& \times \cos \phi \cos n \phi d \phi-\left(\frac{R_{0}}{r}\right)^{n+1} \\
& \left.\times \int_{\Phi(r)}^{\pi / 2} \delta(\phi) l(\phi)^{n+2} \cos \phi \cos n \phi d \phi\right]
\end{aligned}
$$

Here $\Phi(r)$ is the angle between horizontal plane and the position where the radius is $r$. When $r \leq R_{\min }$ we have $\Phi(r)=\pi / 2$, and when $r \geq R_{0}$ we have $\Phi(r)=0$.

\section{TUBE GEOMETRY AND GENERATION OF MULTIPOLE FIELDS}

In this section we investigate the effects of the beam tube geometry on the generation of multipole field. The region $r<R_{\min }$ from the center of the beam tube is the most 
crucial region to keep a high field homogeneity for good particle beam dynamics. We find that the integrated part of Eq. (5) is equal to the normal multipole coefficient $b_{n}$, namely,

$$
b_{n}=\int_{0}^{\pi / 2} \frac{\delta(\phi)}{l(\phi)^{n-2}} \cos \phi \cos n \phi d \phi .
$$

This equation shows how sensitively the beam tube shell geometry parameters $\delta$ and $l$ will affect the field harmonics. A modification of geometric values $\delta$ and $l$ as a function of $\phi$ enables us to eliminate the harmonic of order $n$. As mentioned earlier, typically used beam tubes in accelerators have circular, elliptical, or rectangular cross sections. In the following, we investigate these three cases mathematically for the generation of dominant multipoles, and describe a possible procedure to avoid the generation of higher field harmonics.

\section{A. Circular cross section}

Since $\delta(\phi)$ and $l(\phi)$ are constant in a round beam tube, Eq. (7) has orthogonality for $n>1$ which makes $b_{n}=0$. For $n=1$, Eq. (5) reduces to

$$
B_{\theta}(\theta)=-2 R_{0} D_{0}\left(\frac{\mu_{0} \dot{B}}{\pi \rho}\right) \cos \theta .
$$

As expected, this dipole field is linearly proportional to the thickness $D_{0}$ and radius $R_{0}$ of the beam tube, and is independent of $r$.

\section{B. Elliptical cross section}

In contrast to the circular shape, $R(\phi)$ of an ellipse is not constant. Therefore, in addition to dipole components, we also assume the presence of higher multipoles. A cross sectional shape of a typical elliptical tube is shown in Fig. 1. Using Eq. (5), Fig. 2 shows the corresponding contour field profiles up to decapole. The decapole component has negligibly small values compared to the dipole and sextupole components. In order to compare the multipoles of each order $n$, normal multipole coefficient $b_{n}$ is

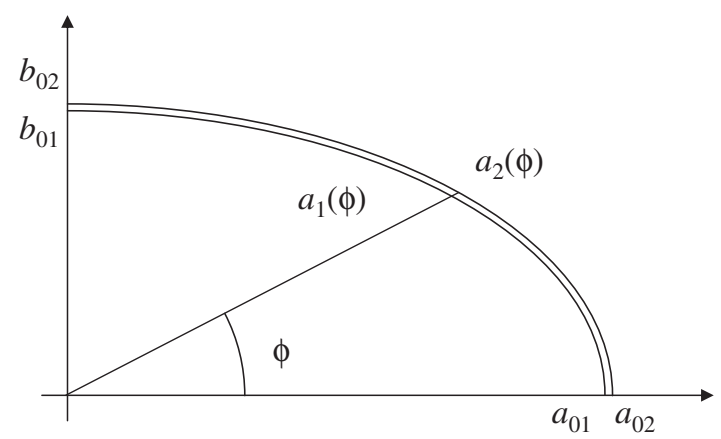

FIG. 1. Cross-sectional view of symmetric on quarter elliptical beam tube. The $a_{1}(\phi), a_{2}(\phi)$ are inner and outer radii. The thicknesses on the horizontal and vertical axes are equal: $\left(a_{02}-a_{01}\right)=$ $\left(b_{02}-b_{01}\right)$. The effective radius is defined as $R(\phi)=\left[a_{1}(\phi)+\right.$ $\left.a_{2}(\phi)\right] / 2$ and generalized thickness $D(\phi)=a_{2}(\phi)-a_{1}(\phi)$.

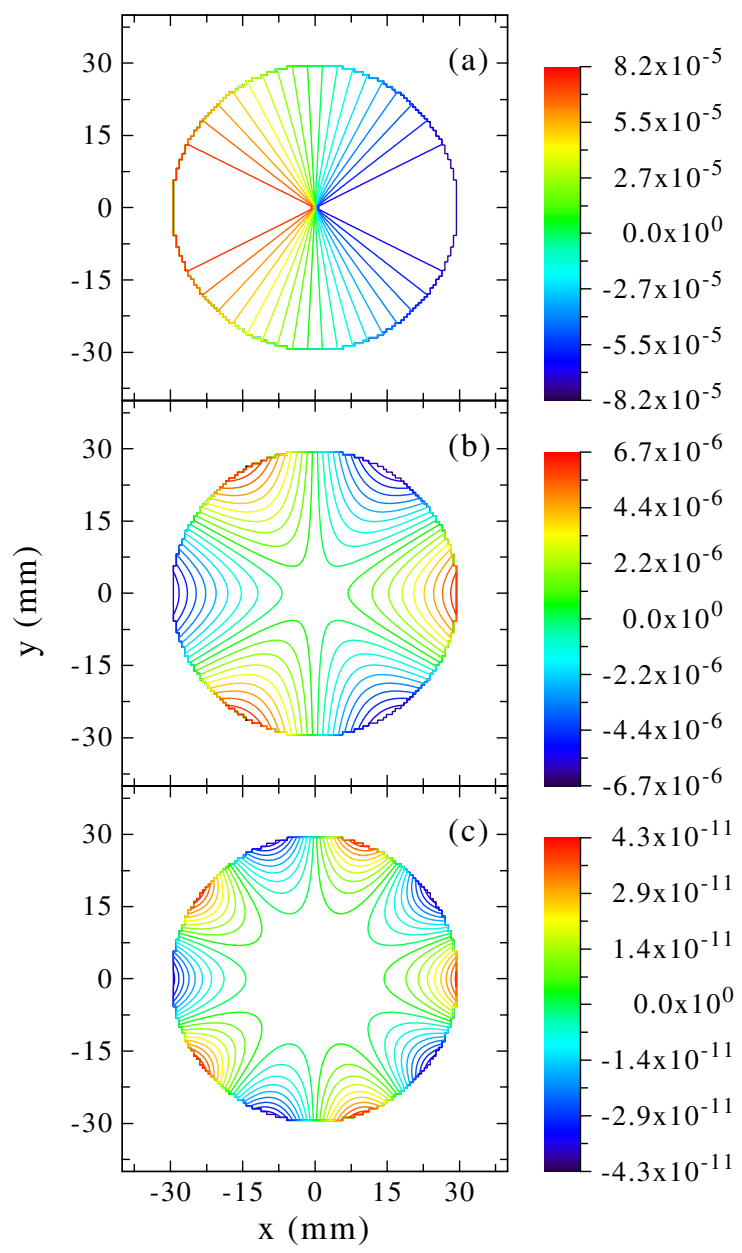

FIG. 2. Harmonic field $B_{\theta}$ (T) profiles up to $n=5$ in the region of $r \leq R_{\min }$ by Eq. (5) with geometry of Fig. 1. (a) Dipole $n=1$, (b) sextupole $n=3$, (c) decapole $n=5$.

plotted in Fig. 3. In addition to the dipole component $n=1$, a significant magnitude can be observed for $n=3$. This sextupole component represents the major order of multipole field response from an elliptical beam tube.

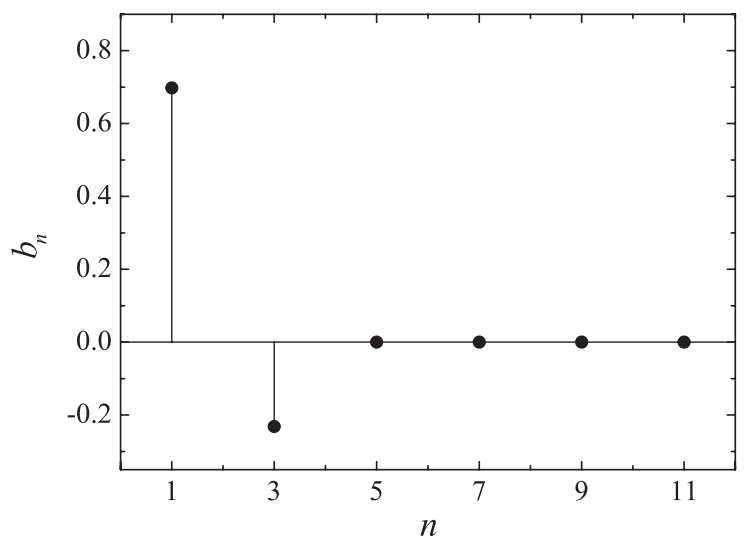

FIG. 3. The normal multipole coefficient $b_{n}$ of an elliptic beam tube shown in Fig. 1. The sextupole component has maximum value in higher orders of $n \geq 3$. 
In order to avoid the generation of a sextupole component, we consider an elliptical beam tube whose generalized thickness $D(\phi)$ varies with angle such that $\delta(\phi)=l(\phi)$. This is equivalent to $D(\phi)=\gamma R(\phi)$ with constant parameter $\gamma$. Hence, $D(\phi)$ changes with effective radius. Figure 4 shows a schematic of the tube cross section with thickness gradient. Thus, the thickness on the major axis is greater than on the minor axis. Using this condition, the coefficient $b_{n}$ takes on the following form:

$$
b_{n}=\int_{0}^{\pi / 2} \frac{1}{l(\phi)^{n-3}} \cos \phi \cos n \phi d \phi .
$$

From Eq. (9) follows that $l(\phi)$ has a degree of $n-3$. Therefore, for harmonic order of $n=3, l(\phi)^{n-3}$ is constant in the angular range from 0 to $\pi / 2$. The multipole coefficient $b_{3}$ is then zero. The calculated normal multipole coefficient $b_{n}$ of each order $n$ is shown in Fig. 5. As expected, only a significant dipole component can be observed. The sextupole component, however, disappears completely in this beam tube geometry. Other higher multipoles have negligibly small values.

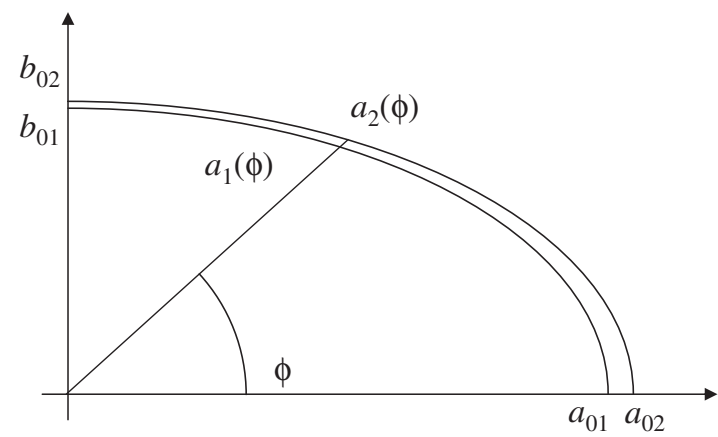

FIG. 4. Elliptic cross section with thickness gradient. The thickness is a function of effective radius $D(\phi)=\gamma R(\phi)^{n-2}$. The equivalent normalized expression is $\delta(\phi)=l(\phi)^{n-2}$. This model enables the deletion of selected harmonics of order $n$.

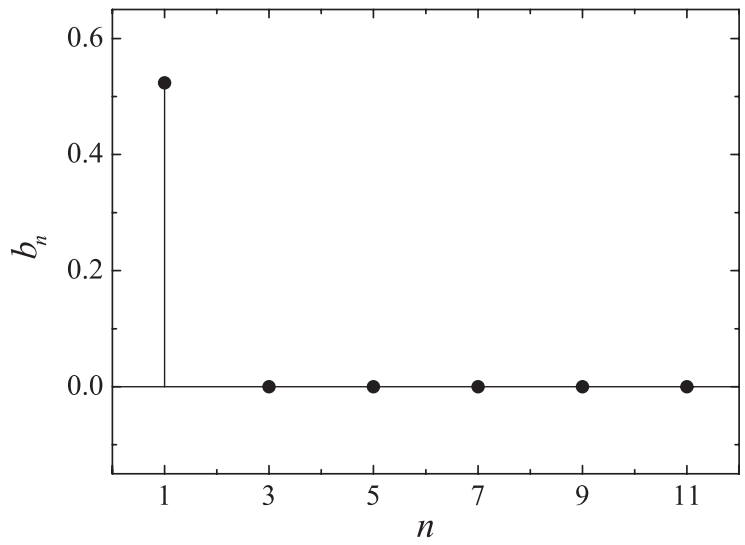

FIG. 5. The normal multipole coefficient $b_{n}$ of an elliptic beam tube with geometry shown in Fig. 4 . The sextupole component is zero after changing the thickness with $\delta(\phi)=l(\phi)$.

\section{Rectangular cross section}

Figure 6 shows the cross section of a beam tube with rectangular shape. The top, bottom, and side walls are each of constant thickness perpendicular to the surface. Hereby, the ratio of the two thicknesses is the ratio of the beam tube dimensions. The bottom and top walls are assumed to be thinner than the side walls as shown in Fig. 6. With this geometry, we easily find the $l(\phi)$ and $\delta(\phi)$ are equal as follows:

$$
l(\phi)=\delta(\phi)=\left\{\begin{array}{ll}
\frac{\cos \phi_{0}}{\cos \phi} & \phi \leq \phi_{0} \\
\frac{\sin \phi_{0}}{\sin \phi} & \phi \geq \phi_{0}
\end{array} .\right.
$$

We see that Eq. (10) has the same geometric condition of an elliptical beam tube with a thickness gradient. Therefore, it is easy to predict that the sextupole will not appear in a rectangular beam tube that has the wall thickness ratio as shown in Fig. 6. The corresponding coefficient $b_{n}$ is

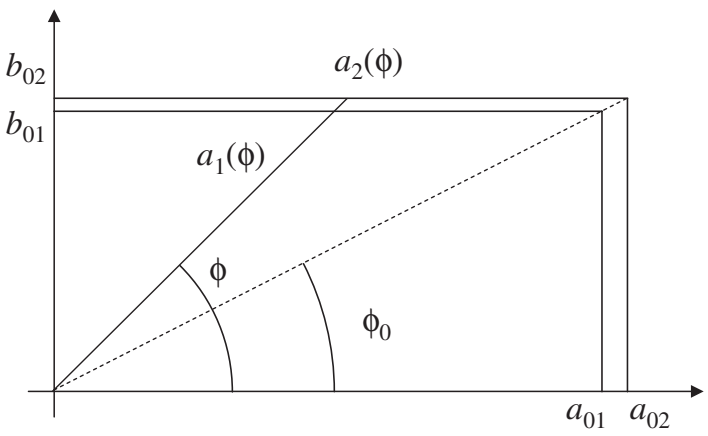

FIG. 6. Cross-sectional view of rectangular beam tube. The horizontal and vertical conducting plates have different wall thicknesses to fit each contact at $\phi_{0}$.

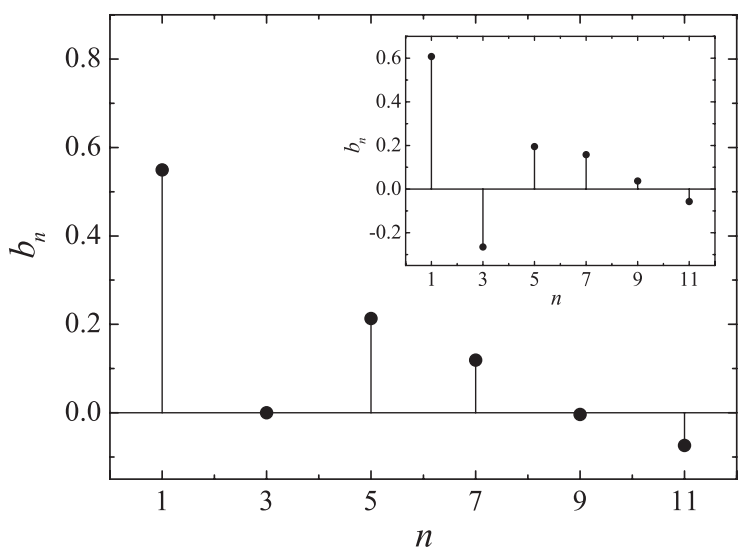

FIG. 7. Corresponding normal multipole coefficients $b_{n}$ of rectangular beam tube shown in Fig. 6. Even though the sextupole component is successfully removed, other multipoles still show noticeable magnitude compared to the dipole value. The inset shows the case of horizontal and vertical plates that have the same chamber wall thickness. It shows that all multipole responses are considerably higher than that of an elliptic beam tube. 


$$
\begin{aligned}
b_{n}= & \int_{0}^{\phi_{0}}\left(\frac{\cos \phi}{\cos \phi_{0}}\right)^{n-3} \cos \phi \cos n \phi d \phi \\
& +\int_{\phi_{0}}^{\pi / 2}\left(\frac{\sin \phi}{\sin \phi_{0}}\right)^{n-3} \cos \phi \cos n \phi d \phi .
\end{aligned}
$$

The multipole coefficient $b_{n}$ needs now to be divided into two integration regions: the vertical and horizontal planes. However, it can be combined when $n=3$. The calculated values of $b_{n}$ is shown in Fig. 7. As expected, the sextupole component $n=3$ is zero, though, higher harmonics still have noticeable values compared to the case of an elliptic beam tube.

For comparison, the plot inset in Fig. 7 shows normal multipole coefficients $b_{n}$ of a rectangular beam tube whose top, bottom, and side wall thicknesses are equal. In this case, a significant sextupole component can be observed, considering there is no orthogonality of the integration, all odd-number harmonic responses appear.

\section{CONCLUSION}

We have studied the relationship between the eddy current-induced field and beam tube geometry in a dipole magnetic field. We analytically described the multipole fields in terms of beam tube geometry and applied our model to commonly used beam tube shapes. A circular beam tube generates only a dipole eddy current-induced field. Furthermore, we found out that a tube with elliptical cross section produces noticeable sextupole components among other higher harmonics of order $n \geq 3$. However, it seems possible to eliminate the sextupole component by using an elliptical beam tube when its wall thickness is angle dependent. Even higher multipoles can be reduced to negligible magnitude by this simple shape modification. In addition, we have demonstrated that a rectangular tube whose bottom and top walls are thinner than its side walls, when applying the correct ratio, does not induce a sextupole field component in a ramping dipole field. Nevertheless, other higher multipoles showed noticeable magnitudes. The method to avoid multipole components of $n \geq 3$ can be summarized with the relation of normalized thickness and normalized radius, $\delta(\phi)=l(\phi)^{n-2}$. Based on this relation, it should be possible to avoid selected multipole components by a modification of the tube wall thickness.

[1] S. Casalbuoni, A. Grau, M. Hagelstein, R. Rossmanith, F. Zimmermann, B. Kostka, E. Mashkina, E. Steffens, A. Bernhard, D. Wollmann, and T. Baumbach, Phys. Rev. ST Accel. Beams 10, 093202 (2007).

[2] H. D. Gersem, S. Koch, S. Y. Shim, E. Fischer, G. Moritz, and T. Weiland, IEEE Trans. Appl. Supercond. 18, 1613 (2008).

[3] S. Koch, H.D. Gersem, E. Fischer, G. Moritz, and T. Weiland, IEEE Trans. Appl. Supercond. 16, 334 (2006).

[4] E. Fischer, P. Schnizer, P. Akishin, R. Kurnyshov, A. Mierau, B. Schnizer, S. Y. Shim, and P. Sherbakov, IEEE Trans. Appl. Supercond. 20, 218 (2010).

[5] A. Jain, J. Escallier, G. Gantetis, W. Louie, A. Marone, R. Thomas, and P. Wanderer, IEEE Trans. Appl. Supercond. 16, 1370 (2006).

[6] G. Sinha and S. S. Prabhu, Phys. Rev. ST Accel. Beams 14, 062401 (2011).

[7] N. J. Siakavellas, IEEE Trans. Magn. 33, 2245 (1997).

[8] S. Y. Lee, Nucl. Instrum. Methods Phys. Res., Sect. A 300, 151 (1991).

[9] N.S. Sereno and S.H. Kim, Report No. ANL/APS/LS302, 2003.

[10] K.-H. Mess, P. Schmüser, and S. Wolff, Superconducting Accelerator Magnets (World Scientific, Singapore, 1996).

[11] K. Wille, The Physics of Particle Accelerators (Oxford University Press, Oxford, 2009). 